\title{
Muographic data analysis method for medium-sized rock overburden inspections
}

\author{
Hiroyuki K. M. Tanaka ${ }^{1}$ and Michinori Ohshiro ${ }^{2}$ \\ ${ }^{1}$ Earthquake Research Institute, The University of Tokyo, 1-1-1 Yayoi, Bunkyo, 113-0032 Tokyo, Japan \\ ${ }^{2}$ Department of History, Komazawa University, 1-23-1 Komazawa, Setagaya, 154-8525 Tokyo, Japan \\ Correspondence to: Hiroyuki K. M. Tanaka (ht@ riken.jp)
}

Received: 2 March 2016 - Published in Geosci. Instrum. Method. Data Syst. Discuss.: 15 March 2016

Revised: 9 August 2016 - Accepted: 20 August 2016 - Published: 21 September 2016

\begin{abstract}
Muographic measurements of rock overburdens are of particular interest because they can be applied to natural resources and undiscovered cave explorations, and even to searching for hidden chambers in historic architectural structures. In order to derive the absolute density distribution of the overburden, we conventionally needed to know accurate information about the measurement conditions, e.g., the detector's geometrical acceptance, detection efficiency, and measurement time, in order to derive the absolute value of the transmitted muon flux. However, in many cases, it is not a simple task to accurately gauge such conditions. Open-sky muon data taken with the same detector are useful as reference data to cancel these factors; however, if the detector is not transportable, this data taking method is not feasible. In this work, we found that the transmitted muon flux will follow a simple function of the areal density along the muon path as long as the incident muon energies are below a few hundred GeV. Based on this finding, we proposed a simple analysis method that does not require detailed knowledge of the detector's conditions by combining the independently measured density information for the partial volume of the target. We anticipate that this simple method is applicable to future muographic measurements of rock overburdens.
\end{abstract}

\section{Introduction}

Earth's subsurface density structures have been extensively measured with muography in the last decade. High-energy muons originating from cosmic rays have strong penetrative power to resolve the density distribution of gigantic objects. The muography technique was first applied by Alvarez et al. (1970) as a method to search for hidden chambers inside the second pyramid of Chephren. Muon detectors installed inside the Belzoni chamber recorded the arrival angles of comic ray muons after they had penetrated the pyramid and reached the chamber. Muography utilizes the natural tendency of high-energy muons to penetrate gigantic materials in the same way that the $\mathrm{x}$-ray does; however, while the $\mathrm{x}$-ray is applicable only to objects of up to $1 \mathrm{~m}$ in thickness, muography visualizes density distributions inside much larger objects. Since George (1955) first proposed and implemented densimetric measurements of the rock overburden above a gallery tunnel of the Snowy Mountains Hydro Electric Authority in Australia with cosmic-ray muons, particle detection techniques had been developed to the extent that 12 years later, Alvarez's group could successfully apply spark chambers with digital readout units to the technique of muography. After analyzing data collected during several months of operation, they concluded that areal density of the pyramid was measured with a precision of $2 \%$ along the muon paths that penetrated over $100 \mathrm{~m}$ through limestone in the pyramid. This pioneering experiment was a crucial step that eventually led to recent muographic experiments that explored inside volcanoes (Tanaka et al., 2007, 2008, 2009; Lesparre et al., 2012; Cârloganu et al., 2012; Carbone et al., 2013; Kusagaya and Tanaka, 2015a, b), industrial plants (Tanaka, 2013; Ambrosino et al., 2015), seismic faults (Tanaka et al., 2011; Tanaka, 2015), and caves (Caffau et al., 1997; Barnaföldi et al., 2012; Oláh et al., 2012).

Cosmic-ray muons are generated in the Earth's atmosphere as secondary cosmic rays and the integrated vertical open-sky muon flux $I_{\mathrm{SKY}}$ is known to be $\sim 70 \mathrm{~m}^{-2} \mathrm{sr}^{-1} \mathrm{~s}^{-1}$. Since the first discovery of muons by Neddermeyer and An- 
derson (1936), the differential flux of these particles has been precisely measured (Olive et al., 2014). The muon flux is reduced after passing through matter. This reduction can be theoretically calculated by integrating the differential flux over the energy range between the cutoff energy, $E_{\mathrm{c}}$, and infinity, where $E_{\mathrm{c}}$ is the minimum energy required for muons to penetrate the target object. $E_{\mathrm{c}}$ can be precisely derived with Monte Carlo simulations, which are based on our knowledge of the standard model of particle physics; therefore, there are almost no uncertainties in these theories (Groom et al., 2001). Once muons are irradiated to the surface of the rock overburden, they are detected and recorded by the detector underneath the overburden to generate the histogram of the number of muon events as a function of the muon's arriving angle.

Muography has the capability to derive an areal density along the muon path. George (1955) compared the muon flux inside and outside of the Guthega-Munyang tunnel, Australia, to calculate the muon's transmission rate, and measured the areal density of the rock overburden of $163 \pm 8 \mathrm{~m}$ water equivalent (mw.e.), which was consistent with the result of the drilling and sampling at the same site: $175 \pm 6 \mathrm{~m}$ w.e. However, the open-sky muon counts are not always available as reference data, particularly when the observation system is not transportable. For example, Alvarez et al. (1970) constructed their observation system inside the Belzoni chamber of the Chephren Pyramid. Their entire system weighed more than 10 ton, and thus it was not realistic to transport the system to the outside of the pyramid to take the reference data without changing the configuration. In this work, we proposed a new method of muographic data analysis for deriving the density distribution of the middle-scale rock overburden (up to a few hundred meters in thickness). With our proposed technique, by combining the value of the density independently measured for a partial volume of the target, we can derive the areal density along the muon path without detailed knowledge about the muon detector. In this paper, we formulated this method and re-analyzed the data presented by Alvarez et al. (1970), Caffau et al. (1997), and Liu et al. (2012) as examples in order to test the method.

\section{Principle}

\subsection{Cosmic-ray muons}

High-energy muons are produced when primary cosmic rays interact with nuclei in the Earth's atmosphere. The energy spectrum of the primary cosmic ray is expressed with the following power law that has an index of $-2.7(\gamma=1.7)$ and is almost constant with energies up to $10^{6} \mathrm{GeV}$.

$$
\frac{\mathrm{d} N}{\mathrm{~d} E}=A E^{-(\gamma+1)}
$$

Consequently, the muon spectrum also closely obeys the power law as the secondary particles of the primaries. Up to this date, several authors have derived the analytical expressions for the differential atmospheric muon spectra in the reaction between primaries and atmospheric nuclei (Bull et al., 1965; Matsuno et al., 1984; Bugaev et al., 1998; Gaisser and Stanev, 2008). The parameters in their models have been adjusted by comparing these with the observed spectrum of muons taken from experiments made at sea level (Jokisch et al., 1979; Matsuno et al., 1984; Allkofer et al., 1985; Haino et al., 2004; Achard et al., 2004). The integrated vertical muon flux is proportional to $E^{-2.2}$ in the energy region between 50 and $200 \mathrm{GeV}$.

The geomagnetic deflection depends on the geomagnetic latitude, and has a significant effect on the muon flux with energies up to $10 \mathrm{GeV}$ (Haeshim and Bludman, 1988), while Hansen et al. (2005) reported that the east-west effect is negligible in the vertical cosmic-ray muon flux. Kamiya et al. (1976) reported that the muon charge ratio measured by the MUTRON spectrometer had to be modified with correction factors of 1.35 and 0.75 for muons in the N-W and SE directions, respectively, indicating a strong geomagnetic effect even on high-energy horizontal muons.

\subsection{Muon range}

The processes through which muons interact with matter can be divided into two types: continuous and stochastic. The rate of the muon's energy loss through matter is expressed by

$-\frac{\mathrm{d} E}{\mathrm{~d} X}=a(E)+b(E) E$,

where $E$ is the muon's energy and $X$ is an areal density along the muon path. $a(E)$ is the energy loss caused by the continuous process, and $b(E) E$ is the energy loss caused by the stochastic process.

Via the continuous process, the muon has frequent encounters with atoms, each losing a very small fraction of its energy via the ionization process. Fluctuations in range arise from stochastic processes: bremsstrahlung, direct pair production, and photonuclear interactions. In these processes, the muon loses a large but random fraction of its energy. However, if the muon's energy is much lower than the critical energy, $708 \mathrm{GeV}$ in $\mathrm{SiO}_{2}$, the continuous process is the main process in muon's energy loss, and thus muons of a given energy would have almost a unique range. For example, an energy loss rate of $100 \mathrm{GeV}$ muons via the continuous and stochastic processes is 2.46 and $0.11 \mathrm{MeV} \mathrm{cm}^{2} \mathrm{~g}^{-1}$, respectively. The $100 \mathrm{GeV}$ muons have a range of $406 \mathrm{~m}$ w.e.

The cutoff energy $\left(E_{\mathrm{c}}\right)$ is defined as the minimum energy that a muon can penetrate for a given areal density of rock $(X)$ and is derived by finding the value at which the muon's continuous slowing down approximation (CSDA) range matches $X$ (Fig. 3). The $E_{\mathrm{c}}$ solely depends on an areal density along the muon path in matter, and its mate- 
rial dependence is small. In order to derive the muon flux after passing through matter, the zenith-angular dependence of the muon flux and the continuous slowing down approximation (CSDA) range (Groom et al., 2001) are utilized. The CSDA range is derived by integrating Eq. (2) over a muon's energy $E$ within a range between 0 and the incident energy $E_{0}$. Inversely, once an areal density is determined along the muon paths, the minimum energy that a muon can penetrate through the path is uniquely determined if the muon's energy is much lower than the critical energy.

\section{Results}

In this work, we modeled muographic observations of the rock overburden. We divided the overburden into two horizontal layers (Layer 0 and Layer 1). The muon detector was assumed to be located underneath this overburden. The areal density was then calculated by assuming a uniform density along the muon paths and multiplying it by the geometrically exploited muon path lengths as follows:

$X=\rho_{0} \times\left\langle\ell_{0}\right\rangle+\rho_{1} \times\left\langle\ell_{1}\right\rangle$,

where $\rho_{0}$ and $\rho_{1}$ are assumed densities for Layer 0 and Layer 1 , respectively. Both $\left\langle\ell_{0}\right\rangle$ and $\left\langle\ell_{1}\right\rangle$ are the path lengths averaged over the elevation angle $(\theta)$ and azimuth angle $(\phi)$, according to an angular resolution of the muogram when it is generated. Most muons traverse matter in a linear trajectory. Therefore, the path length of muons can be precisely determined by reading the outer geometry of the target volume. To calculate the CSDA range, the muon's ionization, bremsstrahlung, direct pair production and photonuclear processes are considered. In Fig. 1, the CSDA range of muons in $\mathrm{SiO}_{2}$ is shown. The Monte Carlo calculation results are based on the publication by Groom et al. (2001).

In order to derive the transmitted muon intensity ( $I$ ) (the muon flux after passing through the overburden), the number of muon events counted in each histogram bin $(N)$ is divided by the detector's active area $(S)$ detection efficiency $\left(A_{\text {eff }}\right)$ solid angle $(\Omega)$ and measurement time $(t) . I$ is compared with the theoretical muon intensity $(i)$ to derive the areal density along the muon path $(X)$, where $i$ is calculated by integrating the muon energy spectrum over a range between the muon's cutoff energy $\left(E_{\mathrm{c}}\right)$ and infinity. In this work, we proposed the flux ratio $I_{0} / I_{1}$ to cancel $S, A_{\text {eff }}, \Omega$, and $t$, where $I_{0}$ and $I_{1}$ are the measured intensity after passing through Layer 0 and Layer 1, respectively.

When the muon's energy is less than $100 \mathrm{GeV}$, corresponding to a muon range of $400 \mathrm{~m}$ w.e., the contribution of the stochastic energy loss $(b(E) E)$ is less than $10 \%$ to the total energy loss. Furthermore, the energy loss rate due to the ionization process $(a(E))$ does not have a strong energy dependence. For example, $a(E)=2.3 \mathrm{MeV} \mathrm{cm}^{2} \mathrm{~g}^{-1}$ when the muon energy is $20 \mathrm{GeV}$ (which corresponds to the muon range of $90 \mathrm{~m}$ w.e.), and it becomes $2.5 \mathrm{MeV} \mathrm{cm}^{2} \mathrm{~g}^{-1}$

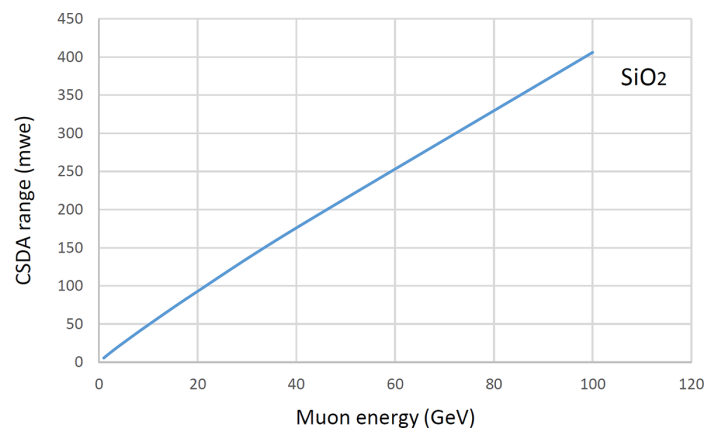

Figure 1. CSDA range of muons in units of meter water equivalent in $\mathrm{SiO}_{2}$.

when muon energy is $200 \mathrm{GeV}$ (which corresponds to the muon range of $740 \mathrm{~m}$ w.e.). Therefore, we can expect that the CSDA range can be fit by a near-linear function within this energy range.

In order to confirm this assumption, the CSDA range reported by Groom et al. (2001) was fit by the following equation:

$E_{\mathrm{c}}=A X^{\varepsilon}$,

where $X$ is the CSDA range. $A$ and $\varepsilon$ are parameters. $E_{\mathrm{c}}$ is measured in $\mathrm{GeV}$ and $X$ in meter water equivalent ( $\mathrm{m}$ w.e.). The results of the fitting five data points $(40,80,100,140$, and $200 \mathrm{GeV}$ ) are shown in Eq. (5).

$A=0.1224$,

$\varepsilon=1.1176$.

The fitting accuracy was about $0.7 \%$. Within this energy range the muon range varies from 180 to $740 \mathrm{~m}$ w.e. As can be seen in Eq. (5b), the cutoff energy $\left(E_{\mathrm{c}}\right)$ has an almost linear relationship with the areal density. Therefore, we approximated the muon flux ratio $\left(I_{0} / I_{1}\right)$ as follows:

$\frac{I_{0}}{I_{1}} \approx\left(\frac{\left\langle X_{0}\right\rangle}{\left\langle X_{1}\right\rangle}\right)^{-2.2}$,

where $I_{0}$ and $I_{1}$ is the transmitted muon flux after passing through Layer 0 and Layer 1, respectively. $\left\langle X_{0}\right\rangle$ and $\left\langle X_{1}\right\rangle$ are averaged areal density along the muon paths for Layer 0 and Layer 1, respectively. Equation (6) indicates that the muon flux ratio only depends on the ratio of the areal density. As a result, if we know the density of Layer $0\left(\rho_{0}\right)$, we can uniquely derive the average density of Layer $1\left(\rho_{1}\right)$ by dividing $\left\langle X_{1}\right\rangle$ by the average thickness of Layer $1\left(\left\langle\ell_{1}\right\rangle\right)$, and vice versa. Equations (6) indicates that we can derive these densities without knowing the active area $(S)$, detection efficiency $\left(A_{\text {eff }}\right)$, and solid angle $(\Omega)$ of the detector. By taking this ratio, the factor $(A)$ in Eq. (1) is also cancelled, and only the index of the integrated muon flux is required for the calculation because the index of the muon spectrum power law 
does not vary in this energy range. An index of -2.2 is almost constant within the zenith angular range between 0 and $50^{\circ}$ (Haino et al., 2004; Achard et al., 2004). However, if the areal density of the overburden exceeds $10^{3} \mathrm{~m}$ w.e., the stochastic process becomes dominant in the muon's energy loss, and thus the index $\varepsilon$ in Eq. (4) varies as a function of energy, and thus this simple formulation cannot be used.

If the muon counts $(N)$ contain background events $(\delta n)$, i.e., $N_{0}=n_{0}+\delta n$ and $N_{1}=n_{1}+\delta n$, the muon count ratio $N_{0} / N_{1}$ will not be equal to the actual flux ratio $I_{0} / I_{1}$, where $n_{0}$ and $n_{1}$ are the number of events without background events, respectively. However, we can reasonably assume that $n \gg \delta n$ when the rock overburden above the detector is thicker than $20 \mathrm{~m}$. This thickness is equivalent to 100 times longer than the electron's radiation length in matter. Also, the hadron's interaction length is up to $100 \mathrm{~g} \mathrm{~cm}^{-2}$, which is 50 times shorter than this thickness. Therefore, we can expect that electromagnetic and hadronic components would be effectively removed by the time they reach the detector. This assumption is supported by a result obtained in the similar experiment in 1955. George (1955) installed a Geiger counter in the Guthega-Munyang tunnel, where the rock overburden was previously measured to be an areal density of $175 \pm 6 \mathrm{~m}$ w.e. with the drilling and sampling method. He simply compared the counting rate measured inside and outside the tunnel without any background treatments to obtain an areal density of $163 \pm 8 \mathrm{~m}$ w.e. that is in agreement with the muographically derived areal density. Therefore, it is reasonable for us to approximate Eq. (6) as

$$
\frac{N_{0}}{N_{1}} \approx\left(\frac{\left\langle X_{0}\right\rangle}{\left\langle X_{1}\right\rangle}\right)^{-2.2}
$$

as long as the overburden thickness exceeds $20 \mathrm{~m}$. Figure 2 shows the scheme of the muographic observation of the rock overburden that is divided into two horizontal layers.

\section{Discussion}

In this section, we examined our technique by applying it to three different kinds of targets: the Pyramid of Chephren in Egypt, the limestone cave in Italy called Grotta Gigante, and the Price 5 deposit at the Myra Falls mine, Canada. For all of the targets, the muography data were collected in the past and had already been published. These data were used for testing our technique.

\subsection{Case study 1: the Pyramid of Chephren}

The pyramids of Giza are not geological products. However, their sizes are remarkably large for man-made architecture, and the observational configuration of muography performed by Alvarez et al. (1970) was essentially the same as other types of muography performed inside tunnels to measure the rock overburdens (Oláh, 2012; Caffau et al., 1997;

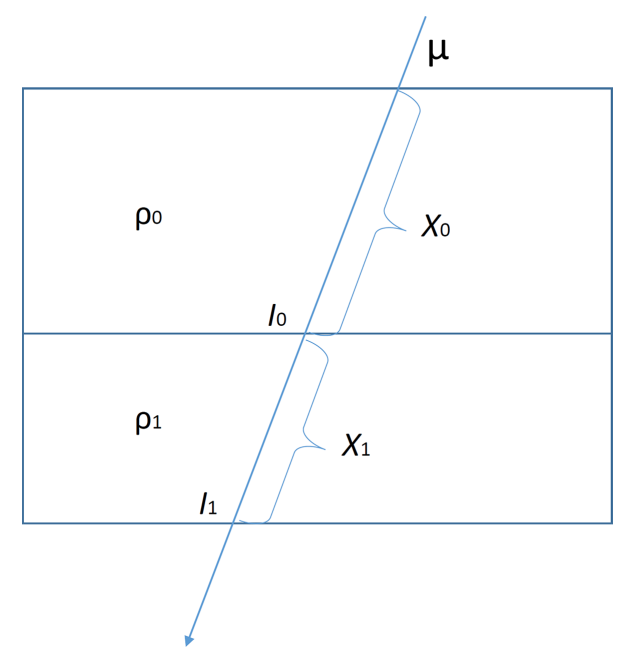

Figure 2. Scheme of the muographic observation of the rock overburden. A Greek letter $\mu$ denotes the incident muons. The rock overburden is divided into two horizontal layers with a uniform density of $\rho_{0}$ and $\rho_{1}$, respectively. $I_{0}$ and $I_{1}$ are the expected numbers of muon intensity, respectively, at the bottom of each layer.

George, 1955). The Pyramid of Chephren offers us a unique target volume to test our technique for the following reasons: (A) the geometrical shape of the pyramid is much simpler than regular geological targets and its topographic features have been studied with aerial surveys (Alvarez et al., 1970); (B) Gerald Lynch did a direct measurement of a rock piece exploited from the surface of the Chephron Pyramid in 1968, and derived a density of $1.8 \mathrm{~g} \mathrm{~cm}^{-3}$ (Alvarez, 1987); (C) the subsurface structure can be directly observed in the partial cross section located near the top of the Pyramid of Chephren; and (D) the Pyramid of Chephren is the only pyramid in which muography surveys were performed by Alvarez et al. in 1970 .

The Pyramid of Chephren is the second largest of the Giza pyramids and the tomb of the Fourth-Dynasty pharaoh Chephren (2558-2532 BC) It is located $20 \mathrm{~km}$ southwest of central Cairo (Fig. 1). After construction, the height was probably $143.87 \mathrm{~m}$; however, now the base length measures $215.5 \mathrm{~m}$ and the height measures $136.4 \mathrm{~m}$. This reduction in height occurred as a result of the loss of the capstone originally located at the apex of the pyramid. A unique feature of the Chephren Pyramid is that the original casing stones made of Tura limestone remain on the upper region of the pyramid (Fig. 2). Below the lower border of the existing casing stones, all casing stones as well as several back stones (those stones located behind the casing stones) have been lost, and as a result the subsurface structure of the pyramid is exposed. This subsurface exposure allows us to see a partial cross section of the interior structure and reveals that both casing stones and back stones are tightly packed without spaces and that one layer of casing stones originally covered the surface of the entire pyramid (Fig. 2b). The pyramid was probably intact until at 
Table 1. Number of muon events collected by Alvarez et al. (1970) in the direction of west to east. The casing stones remain well within an angle range between 72 and $87^{\circ}$. The events are integrated over $24^{\circ}$ wide bands in the north to south direction.

\begin{tabular}{lccccc}
\hline$\theta\left(^{\circ}\right)$ & $72-75$ & $75-78$ & $78-81$ & $81-84$ & $84-87$ \\
$N$ & 10698 & 11019 & 10499 & 11318 & 11900 \\
$N_{1}$ & 11207 & 11746 & 10875 & 11857 & 12367 \\
$N / N_{1}$ & 0.955 & 0.938 & 0.965 & 0.955 & 0.962 \\
\hline
\end{tabular}

least until $100 \mathrm{BC}$, since Diodorus Siculus wrote in that year that "the stones remain to this day still preserving their original position and the entire structure undecayed" (Siculus and Oldfather, 1933). Therefore, trusting this description, we can assert that in the intervening 2100 years, the subsurface materials (casing and back stones) of the Chephren Pyramid were lost. Croci and Biritognolo (2000) reported, based on their close observations, their conclusion that the missing materials had been systematically removed in a manner similar to quarrying techniques.

Two different types of limestone were used to build Chephren's pyramid: Tura and Mokattam. Tura limestone (highquality stone similar to marble) was used for the decorative casing stones. Arnold (1991) reported that the density of limestone used for the casing stones ranges from 2.65 to $2.85 \mathrm{~g} \mathrm{~cm}^{-3}$. Tura limestone is thought to have covered the outside layer of the pyramid and accounted for about $5 \%$ of its entire volume. In comparison to the Tura limestone, the Mokattam limestone is more porous and less dense, and was used for the core of the pyramid. Although the blocks of Mokattam limestone were exploited near the Chephren Pyramid, some of them were transported from a quarry located in southeastern Cairo, Egypt. The geology of this quarry is attributed to the Middle Eocene series. According to Arnold (1991), the average density of limestone used for building the core of the pyramid ranges from 1.7 to $2.6 \mathrm{~g} \mathrm{~cm}^{-3}$. Gerald Lynch did a direct measurement of the rock piece taken from the surface of the Chephron Pyramid in 1968, and derived a density of $1.8 \mathrm{~g} \mathrm{~cm}^{-3}$ (Alvarez, 1987).

Alvarez et al. (1970) collected 650000 muons during their muography experiment in 1968; 100000 of these muons had passed through the upper zone that consists of casing, back stone, and core layers, as defined in Fig. 2. However, there is no description of the measurement time $t$ in Alvarez et al. (1970). This unknown parameter is cancelled if we take the ratio of the number of muon events accumulated in the different histogram bins. The muon counts are statistically sufficient to apply Eq. (7) to compare the subsurface density (the density of the deviation near the apex that consists of casing and back stones) with the pyramid's core density (the average density of the pyramid without this deviation part). For the calculation of $\left\langle\ell_{0}\right\rangle$ and $\left\langle\ell_{1}\right\rangle$, the virtual detector was located $13.5 \mathrm{~m}$ east and $4 \mathrm{~m}$ north of the center on the

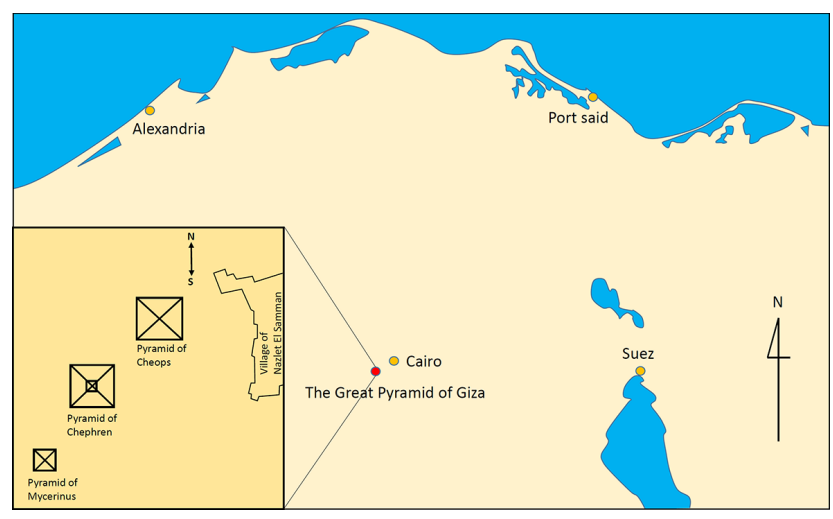

Figure 3. Location of the Great Pyramid of Giza. The inset shows geometrical arrangements of the pyramids of Cheops, Chephren, and Mycerinus.

ground level as described in the report written by Alvarez et al. (1970) (Fig. 2).

In Table 1, we show the number of muon events $(N)$ that Alvarez et al. (1970) collected inside the Belzoni Chamber together with the number of events without casing and back stones $\left(N_{1}\right)$ and their ratio $\left(N / N_{1}\right)$. For this, we employed the value simulated by Alvarez et al. (1970) for the numbers of events without casing and back stones. The events are integrated over $24^{\circ}$ wide bands centered on the middle of the pyramid in the north to south direction. In order to reduce uncertainties arising from irregular casing near the lower border of the upper zone, the analysis was constrained to an angle range between 72 and $87^{\circ}$. As a result, a value of $1.08 \pm 0.1$ was obtained for the ratio of the subsurface density $\left(\rho_{0}\right)$ to the core density $\left(\rho_{1}\right)$. In Table 2 and Fig. 5, the ratio $\rho_{0} / \rho_{1}$ is shown as a function of elevation angle. The ratio does not vary with the muon's arriving angles beyond the error bars associated with these values. In order to derive $\rho_{0} / \rho_{1}$ from $X_{0} / X_{1}, I_{0}(\theta, \varphi)$ and $I_{1}(\theta, \varphi)$ were calculated based on the topography of the pyramid and the detector location, and averaged over the range within an elevation angle range shown in Table 1.

\subsection{Weight of the pyramid}

As an outlet of deriving the density ratio between the subsurface density and the core density of the pyramid, we attempted to derive the total weight of the pyramid. Lehner (2007) measured the thicknesses of the casing stones to be $82,67,45,66,44$, and $45 \mathrm{~cm}$ from the lower edge of the upper zone to the apex. The size of the stones with the exception of those near the lower edge has been calculated to be an average of $53 \mathrm{~cm}$. Therefore, we assumed that a quarter of the volume of the deviation consists of Tura limestone within a density range of $2.75 \pm 0.10 \mathrm{~g} \mathrm{~cm}^{-3}$. The pyramid consists of (A) Tura limestone, (B) back stone, and (C) core stone. A density of (A) is given by Arnold (1991) 
Table 2. Ratio of the average density of the deviation (the part remaining near the apex that consists of casing and back stones) to the density of the pyramid without the deviation part.

\begin{tabular}{lccccc}
\hline$\theta\left(^{\circ}\right)$ & $72-75$ & $75-78$ & $78-81$ & $81-84$ & $84-87$ \\
$\rho_{0} / \rho_{1}$ & $1.08 \pm 0.22$ & $1.57 \pm 0.23$ & $0.90 \pm 0.24$ & $1.19 \pm 0.23$ & $0.95 \pm 0.22$ \\
\hline
\end{tabular}

and a density of (B) $\left(1.8 \mathrm{~g} \mathrm{~cm}^{-3}\right)$ is given by Alvarez (1987). Since (A) remains only at the top of the pyramid, Alvarez's measurement gives the number of muon counts after passing through all of (A), (B) and (C), and through only (C). Since it is reasonable to assume that the density of $(B)$ is the same as that of $(\mathrm{C})$, the density of $(\mathrm{C})$ can be calculated. The back stone density is $1.8 \mathrm{~g} \mathrm{~cm}^{-3}$; the pyramid's core density will be $1.89 \pm 0.20 \mathrm{~g} \mathrm{~cm}^{-3}$, which is in agreement with the back stone density within the statistical error. Since both casing stones and back stones are tightly packed without spaces (at least near the surface), this assumption is reasonable. By considering the total volume of the Chephren Pyramid to be $2211096 \mathrm{~m}^{3}$, the total weight of the pyramid is derived to be $3.98 \times 10^{6} \mathrm{t}$.

A thermal scan technique that provides us with a spatial distribution of subsurface thermal conductivity complements muography technique that gives us an areal density distribution along various muon paths and, therefore, a joint measurement between muography and thermal scans, will provide us with a more comprehensive picture on structural irregularities inside a pyramid.

\subsection{Case study 2: Grotta Gigante}

The area around the natural limestone cave called Grotta Gigante consists of $400 \mathrm{~m}$ thick limestone bedrock including a known aquifer located $250 \mathrm{~m}$ below the ground surface, and a number of branch caves are undiscovered. Karst topography is a unique geological landscape formed by the natural dissolution of soluble bedrock, mostly made up of a thick layer of limestone that is eroded by being dissolved gradually into the underground aquifer. This dissolution is likely to occur in the crack of the ground, and thus the specific part of the ground tends to be eroded and forms a doline (sink hole) on the ground surface and a limestone cave underground. As a result, a complicated cave system that consists of a number of small caves will spread around the region where the large main cave is developed. Many of the caves are not opened to the outside, and therefore it is expected that the unique ecosystem is developed there without being disturbed by exterior biological activities (e.g., Rohwerder et al., 2003).

Caffau et al. (1997) installed their detector at a location of $115 \mathrm{~m}$ below the ground surface in order to map out the areal density of the rock overburden of Grotta Gigante in various directions. The detector was located underneath the region with the presence of the doline on the ground surface. The void associated with the doline exists in the direction of $50^{\circ}$ from the zenith. The typical rock density around the detector was measured to be $2.7 \mathrm{~g} \mathrm{~cm}^{-3}$. The angular resolution of the generated muogram was 10 and $5^{\circ}$ in the azimuthal and elevation direction, respectively. The thickness of the rock overburden in the vertical direction $\left(\left\langle\ell_{0}\right\rangle\right)$ was $20 \mathrm{~m}$; i.e., the corresponding areal density $\left(\left\langle X_{0}\right\rangle\right)$ was $54 \mathrm{~m}$ w.e., and the number of muon events counted in this direction $\left(N_{0}\right)$ was 200. On the other hand, the rock thickness in the direction of $50^{\circ}$ from zenith $\left(\left\langle\ell_{1}\right\rangle\right)$ was $70 \mathrm{~m}$ after reduction of the cave effect, and the corresponding number of muon events $(N)$ was 25 . The muon flux ratio $\left(N_{0} / N_{1}\right)$ can therefore be calculated to be 8 . From Eq. (7), $X_{1} / X_{0}$ is then calculated to be 2.6 , and here we assumed that the vertical muon flux closely matches with the flux in the direction of $50^{\circ}$ from zenith (Haino et al., 2004; Achard et al., 2004). Consequently, the areal density of the overburden in the direction of $50^{\circ}$ is derived to be $140 \mathrm{~m}$ w.e. If we assumed the uniform density of $2.7 \mathrm{~g} \mathrm{~cm}^{-3}$, the overburden thickness in this direction will be $52 \mathrm{~m}$, which is slightly shorter than the expected $\left\langle\ell_{1}\right\rangle$.

Once the muographic anomalies are detected, we can compare them with the gravimetric data. In the area of the Grotta Gigante, the Osservatorio Geofisico Sperimentale (OGS) conducted a gravimetric survey using a LaCoste-Romberg microgravity meter. More than 200 data points were acquired in an area of $\sim 800 \times 650 \mathrm{~m}^{2}$ to map out the gravity anomaly in this area. The region where the muographic anomaly was observed showed a gravity deviation of $0.1 \mathrm{mgal}$ from the value expected from the terrain topography. By combining these data with the gravimetric data, it was revealed that this anomaly came from a red-soil deposit laying beneath the doline, and its volume and total weight were estimated to be $5 \times 10^{3} \mathrm{~m}^{3}$ and $8.5 \times 10^{6} \mathrm{~kg}$, respectively (Caffau et al., 1997).

\subsection{Case study 3: ore body explorations}

This muographic data analysis may also be applied to localization of ore bodies because typically the ore density is 1.4-1.8 times higher than the surrounding medium. Liu et al. (2012) conducted muographic observations in the Price 5 deposit at the Myra Falls mine, Canada, in order to estimate the total weight of the zinc ore body. This target was suitable for the proof of concept trial for muographic mineral explorations because the mine gallery exists at relatively shallow depths from the ground surface and a density map is available based on the diamond drilling results. The averaged ore and the surrounding medium density were, respectively, measured to be 3.2 and $2.7 \mathrm{~g} \mathrm{~cm}^{-3}$ based on the drilling and sampling method prior to their muographic measurements. A 
(a)

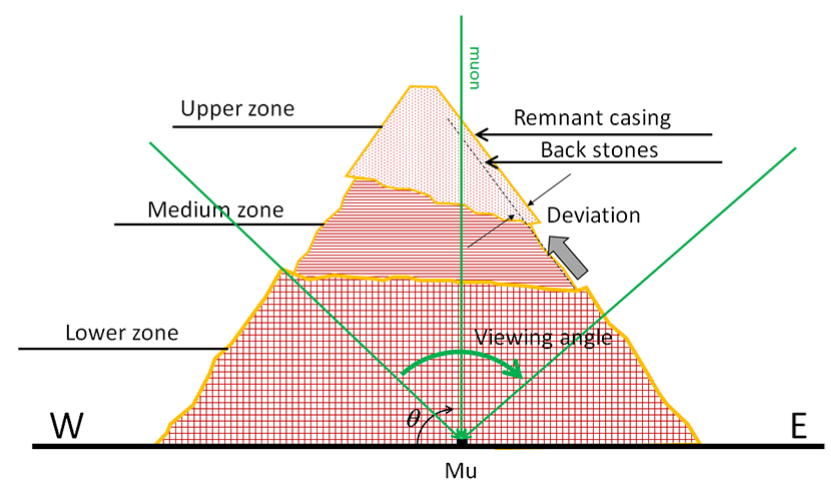

(b)

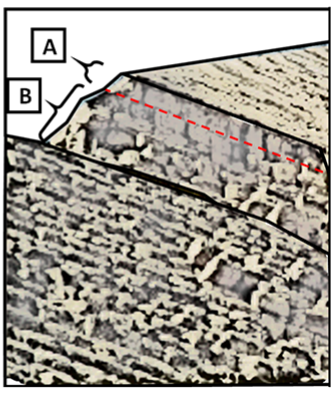

Figure 4. Geometric configuration of muographic measurements performed by Alvarez et al. (1970) along with a view of the south face of the Chephren Pyramid. The viewing angle and the position $(\mathrm{Mu})$ of their apparatus are shown. An elevation angle $(\theta)$ is defined as an angle measured from the west to the east direction. The upper, medium and lower zones were defined based on the different surface conditions that were characterized by Croci and Biritognolo (2000). The region called "deviation" was defined by Alvarez et al. (1970) as distances from the surface of the medium zone. Casing remained on the surface of the upper zone. The grey arrow shows the viewing direction in which we can see the cross-sectional subsurface of the upper zone (a). The illustration of the cross-sectional view contains casing [A] and back stones [B] (b). The deviated region consists of a mixture of the remaining casing and back stones.

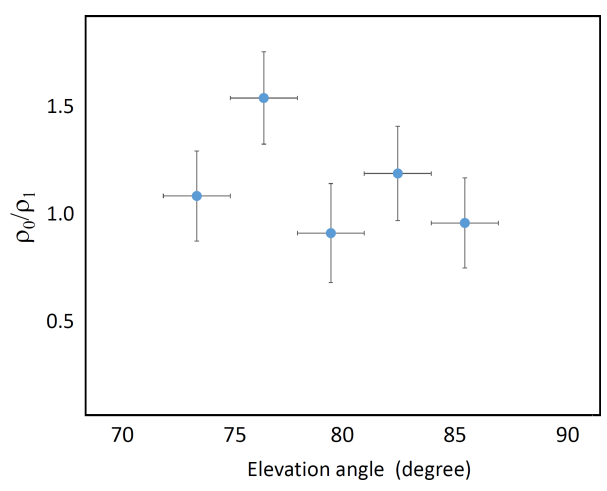

Figure 5. Density ratio of the deviated region that consists of casing and back stones $\left(\rho_{0}\right)$ to the pyramid's core $\left(\rho_{1}\right)$ as a function of an elevation angle $(\theta)$.

$348 \mathrm{~h}$ operation of the detector located underneath the overburden not containing the ore body (Overburden A) collected 5.6. $\times 10^{5}$ muons, while a $283 \mathrm{~h}$ operation underneath the overburden that contains the ore body (Overburden B) collected $1.6 \times 10^{5}$ muons. The muon flux ratio $\left(N_{0} / N_{1}\right)$ can therefore be calculated to be 2.8. The geometrical thickness of Overburden A $\left(\left\langle\ell_{0}\right\rangle\right)$ and Overburden B $\left(\left\langle\ell_{1}\right\rangle\right)$ is, respectively, $\sim 100$ and $\sim 140 \mathrm{~m}$. Likewise, from Eq. (7), $\left(X_{0} / X_{1}\right)^{-1}$ is then calculated to be 1.6. Since the areal density of Overburden $\mathrm{A}$ is calculated to be $\sim 270 \mathrm{~m}$ w.e., the areal density of Overburden B is derived to be $\sim 430 \mathrm{~m}$ w.e.; therefore, the average density is $3.1 \mathrm{~g} \mathrm{~cm}^{-3}$. By inputting the ore density of $3.2 \mathrm{~g} \mathrm{~cm}^{-3}$, the thickness of the ore can be calculated to be $\sim 100 \mathrm{~m}$, which is in agreement with the result from the prior drilling and sampling work. We anticipate that this method will also be applicable to exploring the pyrite-

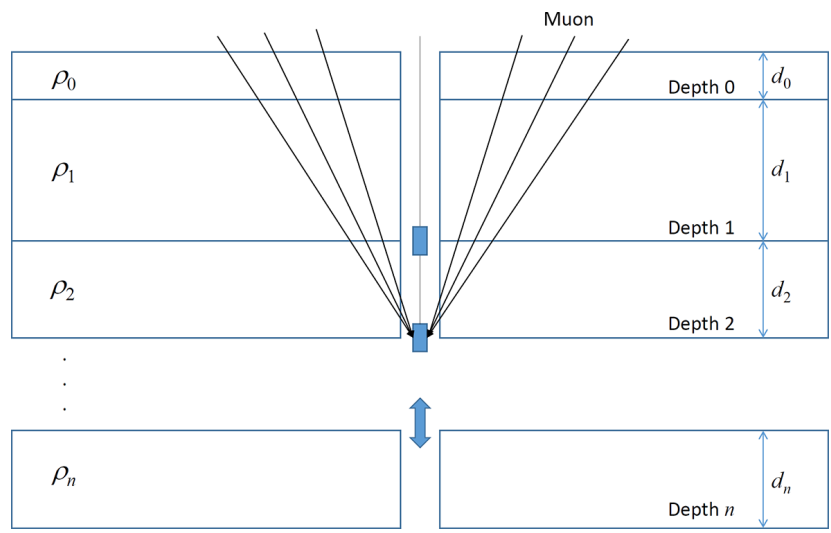

Figure 6. Conceptual drawing of the muographic density derivation by using a borehole. $\rho_{0}, \rho_{1}, \ldots$, and $\rho_{n}$, respectively, denote the average density in Layer 0 , Layer $1, \ldots$, and Layer $n$ with a thickness of $d_{0}, d_{1}, \ldots$, and $d_{n}$.

polymetallic and wolfram deposits of the Greater Caucasus (Eppelbaum and Khesin, 2012).

This technique is applicable to future underground muography observations by utilizing, e.g., a borehole (Fig. 6) . Once we determine the near-surface density $\left(\rho_{0}\right)$ with drilling and sampling methods, the average density of the depth region $\left(d_{1}\right)$ between the core sampling depth $\left(d_{0}\right)$ and the detector location $(d)$ will be measured without requiring knowledge of the active area $(S)$, detection efficiency $\left(A_{\text {eff }}\right)$, and solid angle $(\Omega)$ of the detector. If the measurement time is fixed for each measurement, the ratio of the number of muon events $\left(N_{0} / N_{1}\right)$ counted at depths of Depth 0 and Depth 1 will give the density ratio $\rho_{0} / \rho_{1}$ by considering the muon's path length in each layer, which is proportional to the depths, 
$d_{0}$ and $d_{1}$. Therefore, if we compare the number of muon events measured at different depths, the average densities above the detector locations are derived one after another, and thus the vertical density distribution of the soil will be obtained. If two or more boreholes are available for this kind of measurement, three-dimensional information on the density distribution will be obtained.

\section{Conclusion}

In this work, we evaluated the relationship between the transmitted muon flux and the areal density along the muon path, and found that it has a simple relationship as long as the overburden thickness is thinner than a few hundred meters. Based on this finding, we proposed a simple analysis method to cancel the contributions from the active area $(S)$, detection efficiency $\left(A_{\text {eff }}\right)$, and solid angle $(\Omega)$ of the detector in the generated muogram by combining the independently measured density information for the partial volume of the target.

We showed two examples as possible applications of this analysis method. By re-analyzing the muographic data collected by Alvarez et al. (1970) and combining the surface sampling results with them, we derived the bulk density of the core of the Pyramid of Chephren, Egypt; hence its total weight. By combining the drilling and sampling results taken at the region near Grotta Gigante, Italy, with the muographic data collected by Caffau et al. (1997), we calculated the size of the void associated with the doline caused by karst process. The derived size with our method was consistent with the result obtained with the conventional method, which utilized the absolute value of the transmitted muon flux.

Combining non-muographic densimetric techniques with muography has been shown to be useful for improving the spatial resolution of the density image. We showed in the case study of Groitta Gigante that this simple analysis method could be even more powerful by combining gravimetric measurements to provide useful geological information. Recently, Jourde et al. (2015) showed that gravimetric and muographic joint measurements enhance the resolving power of the technique, in particular, when the muographic measurement is unidirectional. Furthermore, since muographic and gravimetric measurements, respectively, derive horizontally and vertically integrated density, a resolution of the deeper region where muography is not applicable becomes greatly improved by this kind of joint measurement.

The near-surface density is relatively easier to measure, e.g., with a drilling sampling method, in comparison to deeper region densities. Therefore, we anticipate that this technique is useful for future muographic measurements of the rock overburden for the purpose of exploring geological structures that require an accurate density value, for example, surveys of natural resources and mechanical fracture regions of the fault zones.

\section{Data availability}

The data we used have been already published and accessible through Alvarez et al. (1970), Caffau et al. (1997), and Liu et al. (2012).

Edited by: L. Eppelbaum

Reviewed by: two anonymous referees

\section{References}

Achard, P., Adriani, O., Aguilar-Benitez, M., Van den Akker, M., Alcaraz, J., et al.: Studies of hadronic event structure in $\mathrm{e}+\mathrm{e}-$ annihilation from 30 to $209 \mathrm{GeV}$ with the L3 detector, Physics Reports, 399, 71-174, 2004.

Allkofer, O. C., Bella, G., Dau, W. D., Jokisch, H., Klemke, G., Oren, Y., and Uhr, R.: Cosmic ray muon spectra at sea-level up to $10 \mathrm{TeV}$, Nucl. Phys. B, 259, 1-18, 1985.

Alvarez, L. W.: Discovering Alvarez: Selected Works of Luis W. Alvarez with Commentary by His Students and Colleagues, University of Chicago Press, Chicago, 1-282, 1987.

Alvarez, L. W., Anderson, J. A., El Bedwei, F., Burkhard, J., Fakhry, A., Girgis, A., Goneid, A., Hassan, F., Iverson, D., Lynch, G., Miligy, Z., Moussa, A. H., Sharkawi, A., and Yazolino, L.: Search for hidden chambers in the pyramid, Science, 167, 832739, doi:10.1126/science.167.3919.832, 1970.

Ambrosino, F., Bonechi, L., Cimmino, L., D’Alessandro, R., Ireland, D. G., Kaiser, R. B., Mahon, D. F., Mori, N., Noli, P., Saracino, G., Shearer, C., Viliani, L., and Yang, G.: Assessing the feasibility of interrogating nuclear waste storage silos using cosmic-ray muons, J. Instrument., 10, 1-13, 2015.

Arnold, D.: Building in Egypt; Pharaonic Stone Masonry, Oxford University Press, Oxford, 1-316, 1991.

Barnaföldi, G. G., Hamar, G., Melegh, H. G., Oláh, L., Surányi, G., and Varga, D.: Portable Cosmic Muon Telescope for Environmental Applications, Nucl. Instrum. Meth. A, 689, 60-69, 2012.

Bugaev, E. V., Misaki, A., Naumov, V. A., Sinegovskaya, T. S., Sinegovsky, S. I., and Takahash, N.: Atmospheric muon flux at sea level, underground, and underwater, Phys. Rev. D, 58, 054001, doi:10.1103/PhysRevD.58.054001, 1998.

Bull, R., Nash, W. F., and Rustin, B. C.: The Momentum Spectrum and Charge Ratio of I - Mesons at Sea-Level - II, Nuovo Cimento, XLA, 2, 365-384, 1965.

Caffau, E., Coren, F., and Giannini, G.: Underground cosmic-ray measurement for morphological reconstruction of the Grotta Gigante natural cave, Nucl. Instrum. Meth. A, 385, 480-488, doi:10.1016/S0168-9002(96)01041-8, 1997.

Carbone, D., Gibert, D., Marteau, J., Diament, M., Zuccarello, L., and Galichet, E.: An experiment of muon radiography at Mt. Etna (Italy), Geophys. J. Int., 196, 633-643, 2013.

Cârloganu, C., Niess, V., Béné, S., Busato, E., Dupieux, P., Fehr, F., Gay, P., Miallier, D., Vulpescu, B., Boivin, P., Combaret, C., Labazuy, P., Laktineh, I., Lénat, J.-F., Mirabito, L., and Portal, A.: Towards a muon radiography of the Puy de Dôme, Geosci. Instrum. Method. Data Syst., 2, 55-60, doi:10.5194/gi-2-55-2013, 2013.

Croci, G. and Biritognolo, M.: The structural behaviour of the Pyramid of Chephren, Arch 2000, 1, 1-6, 2000. 
Eppelbaum, L. V. and Khesin, B. E.: Geophysical Studies in the Caucasus, Springer, Berlin, p. 411, 2012.

Gaisser, T. and Stanev, T.: Cosmic Rays, Phys. Lett. B, 667, 254260, 2008.

George, E. P.: Cosmic rays measure overburden of tunnel, Commonw. Eng., 1955, 455-457, 1955.

Groom, D. E., Mokhov, N. V., and Striganov, S. I.: Muon stoppingpower and range tables: $10 \mathrm{MeV}-100 \mathrm{TeV}$, At. Data Nucl. Data Tabl., 78, 183-356, 2001.

Haeshim, L. and Bludman, S. A.: Calculation of low-energy atmospheric muon flux, Phys. Rev. D, 38, 2906-2907, 1988.

Haino, S., Sanuki, T., Abe, K., Anraku, K., Asaoka, Y., Fuke, H., Imori, M., Itasaki, A., Maeno, T., Makida, Y., Matsuda, S., Matsui, N., Matsumoto, H., Mitchell, J. W., Moiseev, A. A., Nishimura, J., Nozaki, M., Orito, S., Ormes, J. F., Sasaki, M., Seo, E. S., Shikaze, Y., Streitmatter, R. E., Suzuki, J., Takasugi, Y., Tanaka, K., Tanizaki, K., Yamagami, T., Yamamoto, A., Yamamoto, Y., Yamato, K., Yoshida, T., and Yoshimura, $\mathrm{K}$.: Measurements of primary and atmospheric cosmic-ray spectra with the ESS-TeV spectrometer, Phys. Lett. B, 594, 35-46, doi:10.1016/j.physletb.2004.05.019, 2004.

Hansen, P., Gaisser, T. K., Stanev, T., and Sciutto, S. J.: Influence of the geomagnetic field and of the uncertainties in the primary spectrum on the development of the muon flux in the atmosphere, Phys. Rev. D, 71, 083012, doi:10.1103/PhysRevD.71.083012, 2005.

Jokisch, H., Carstensen, K., Dau, W., Meyer, H., and Allkofer, O.: Cosmic-ray muon spectrum up to $1 \mathrm{TeV}$ at $75^{\circ}$ zenith angle, Phys. Rev. D, 19, 1368-1372, 1979.

Jourde, K., Gibert, D., and Marteau, J.: Improvement of density models of geological structures by fusion of gravity data and cosmic muon radiographies, Geosci. Instrum. Method. Data Syst., 4, 177-188, doi:10.5194/gi-4-177-2015, 2015.

Kamiya, Y., Iida, S., and Shibata, S.: Proceedings of the Asian Cosmic Ray Conference, Hongkong, p. 133, 1976.

Kusagaya, T. and Tanaka, H. K. M.: Muographic imaging with a multi-layered telescope and its application to the study of the subsurface structure of a volcano, Proc. Jpn. Acad. Ser. B, 91, 501-510, 2015a.

Kusagaya, T. and Tanaka, H. K. M.: Development of the very longrange cosmic-ray muon radiographic imaging technique to explore the internal structure of an erupting volcano, Shinmoedake, Japan, Geosci. Instrum. Method. Data Syst., 4, 215-226, doi:10.5194/gi-4-215-2015, 2015 b.

Lehner, M.: Pyramids: Treasures Mysteries and New Discoveries in Egypt, White Star Publisher, 46-59, 2007.

Lesparre, N., Gibert, D., Marteau, J., Komorowski, J.-C., Nicollin, F., and Coutant, O.: Density muon radiography of La Soufriere of Guadeloupe volcano: comparison with geological, electrical resistivity and gravity data, Geophys. J. Int., 190, 1008-1019, 2012.
Liu, Z., Bryman, D., and Bueno, J.: Application of Muon Geotomography to Mineral Exploration, International Workshop on "Muon and Neutrino Radiography 2012", 17-20 April 2012, Clermont-Ferrand, France, 2012.

Matsuno, S., Kajino, F., Kawashima, Y., Kitamura, T., Mitsui, K., Muraki, Y., Ohashi, Y., Okada, A., and Suda, T.: Cosmic-ray muon spectrum up to $20 \mathrm{TeV}$ at $89^{\circ}$ zenith angle, Phys. Rev. D, 29, 1-23, 1984.

Neddermeyer, S. and Anderson, C.: Note on the nature of cosmicray particles, Phys. Rev., 51, 884-886, 1936.

Oláh, L., Barnaföldi, G. G., Hamar, G., Melegh, H. G., Surányi, G., and Varga, D.: CCC-based muon telescope for examination of natural caves, Geosci. Instrum. Method. Data Syst., 1, 229-234, doi:10.5194/gi-1-229-2012, 2012.

Olive, K. A., Agashe, K., Amsler, C., et al.: Review of particle physics, Chin. Phys. C, 38, 090001, doi:10.1088/16741137/38/9/090001, 2014.

Rohwerder, T., Sand, W., and Lascu, C.: Preliminary Evidence for a Sulphur Cycle in Movile Cave, Romania, Acta Biotechnol., 23, 101-107, 2003.

Siculus, D. and Oldfather, C. H.: Diodorus Siculus: Library of History, Books 1-2.34, Loeb Classical Library, 279, 1-498, 1933.

Tanaka, H. K. M.: Development of stroboscopic muography, Geosci. Instrum. Method. Data Syst., 2, 41-45, doi:10.5194/gi2-41-2013, 2013.

Tanaka, H. K. M.: Muographic mapping of the subsurface density structures in Miura, Boso and Izu peninsulas, Japan, Sci. Rep., 5, 1-10, 2015.

Tanaka, H. K. M., Nakano, T., Takahashi, S., Yoshida, J., Takeo, M., Oikawa, J., Ohminato, T., Aoki, Y., Koyama, E., Tsuji, H., and Niwa, K.: High resolution imaging in the inhomogeneous crust with cosmic-ray muon radiography: The density structure below the volcanic crater floor of Mt. Asama, Japan, Earth Planet. Sc. Lett., 263, 104-113, 2007.

Tanaka, H. K. M., Nakano, T., Takahashi, S., Yoshida, J., Takeo, M., Oikawa, J., Ohminato, T., Aoki, Y., Koyama, E., Tsuji, H., Ohshima, H., Maekawa, T., Watanabe, H., and Niwa, K.: Radiographic imaging below a volcanic crater floor with cosmic-ray muons, Am. J. Sci., 308, 843-850, 2008.

Tanaka, H. K. M., Uchida, T., Tanaka, M., Shinohara, H., and Taira, H.: Cosmic-ray muon imaging of magma in a conduit: degassing process of Satsuma-Iwojima Volcano, Japan, Geophys. Res. Lett., 36, L01304, doi:10.1029/2008GL036451, 2009.

Tanaka, H. K. M., Miyajima, H., Kusagaya, T., Taketa, A., Uchida, T., and Tanaka, M.: Cosmic muon imaging of hidden seismic fault zones: Raineater permeation into the mechanical fracture zone in Itoigawa-Shizuoka Tectonic Line, Japan, Earth Planet. Sc. Lett., 306, 156-162, 2011. 